\title{
Proinflammatory Role of Vascular Endothelial Growth Factor in the Pathogenesis of Rheumatoid Arthritis: Prospects for Therapeutic Intervention
}

\author{
Seung-Ah Yoo, Seung-Ki Kwok, and Wan-Uk Kim \\ Division of Rheumatology, Department of Internal Medicine, School of Medicine, Catholic University of Korea, \\ Seoul 137040, South Korea \\ Correspondence should be addressed to Wan-Uk Kim, wan725@catholic.ac.kr
}

Received 18 July 2008; Accepted 1 December 2008

Recommended by Yvette Mandi

Recent experimental and clinical studies have placed new emphasis on the role of angiogenesis in chronic inflammatory disease. Vascular endothelial growth factor (VEGF) and its receptors are the best characterized system in the regulation of rheumatoid arthritis (RA) by angiogenesis. Furthermore, in addition to its angiogenic role, VEGF can act as a direct proinflammatory mediator during the pathogenesis of RA, and protect rheumatoid synoviocytes from apoptosis, which contributes to synovial hyperplasia. Therefore, the developments of synovial inflammation, hyperplasia, and angiogenesis in the joints of RA patients seem to be regulated by a common cue, namely, VEGF. Agents that target VEGF, such as anti-VEGF antibody and aptamer, have yielded promising clinical data in patients with cancer or macular degeneration, and in RA patients, pharmacologic modulations targeting VEGF or its receptor may offer new therapeutic approaches. In this review, the authors integrate current knowledge of VEGF signaling and information on VEGF antagonists gleaned experimentally and place emphasis on the use of synthetic anti-VEGF hexapeptide to prevent VEGF interacting with its receptor.

Copyright (c) 2008 Seung-Ah Yoo et al. This is an open access article distributed under the Creative Commons Attribution License, which permits unrestricted use, distribution, and reproduction in any medium, provided the original work is properly cited.

\section{INTRODUCTION}

The pathology of rheumatoid arthritis (RA) is characterized by the proliferation of synovial cells and angiogenesis, pannus formation. Multiple cell types, including lymphocytes, dendritic cells, macrophages, and synovial fibroblasts, contribute to the chronic inflammatory responses of RA, and comprise a major portion of the invasive pannus [1]. In addition, angiogenesis, the process of new blood vessel formation, is highly active in RA, particularly during the earliest stages of the disease $[2,3]$. Newly formed vessels can maintain the chronic inflammatory state by transporting inflammatory cells to sites of synovitis, and supply nutrients and oxygen to the pannus [2,3]. Angiogenesis is strictly regulated by many inducers and inhibitors, and a number of proangiogenic factors have been suggested to be involved in neovascularization in RA joints. These include acidic and basic fibroblast growth factors, transforming growth factor (TGF) $-\beta$, angiopoietin, and placenta growth factor (PIGF) in addition to vascular endothelial growth factor (VEGF) [2-4].

The final goal of RA treatment is complete disease remission, and not symptomatic relief. At one end of the spectrum of RA treatment outcomes lie a large group of patients who do not respond to single disease-modifying antirheumatic drugs (DMARDs) [5]. Recent clinical trials have suggested that several biologic agents, such as TNF- $\alpha$ blockers, rituximab, abatacept, and anakinra, are effective at retarding joint destruction and at alleviating RA activity $[5,6]$. However, these biologic agents may have serious side effects, such as predispositions to tuberculosis, lymphoma, progressive multifocal leukoencephalopathy, and high cost, which limit their use [7]. It is also a concern that abrupt stoppages or reductions in these treatments may result in a relapse of disease activity. Moreover, the pathology of RA suggests that it is unlikely that a single biologic agent that targets a specific subset of immune cells is capable of effecting cure. 
In this review, we integrate current knowledge concerning how angiogenesis, specifically VEGF, contributes to disease exacerbations in RA. In addition, we present a new therapy for RA based on a synthetic anti-VEGF hexapeptide that specifically targets the interaction between VEGF and its receptor. Prospects for the development of pharmacologic regulators of placental growth factor, which is another angiogenic factor implicated in the pathogenesis of RA, also are discussed.

\section{PROINFLAMMATORY AND ANTIAPOPTOTIC ROLES OF VEGF IN THE PATHOGENESIS OF RA}

VEGF is a dimeric glycoprotein that induces the proliferation and migration of endothelial cells to form new blood vessels, and which increases vascular permeability. VEGF plays important roles during wound healing, embryonic development, the growths of certain solid tumors, and during ascites formation [8]. Several recent reports have demonstrated that VEGF is also implicated in the pathogenesis of RA. Smoking has been recognized as a significant environmental risk factor in RA [9]. Numerous links have been found between cigarette smoking and VEGF [10-12]. VEGF in synovial fluids is significantly more increased in RA than in osteoarthritis $[2,13,14]$, and serum levels of VEGF correlate well with RA disease activity, particularly with swollen joint counts [13]. VEGF protein and mRNA are expressed by synovial macrophages and synovial fibroblasts in the synovial tissues of RA patients, and cultured synovial cells are able to secrete VEGF under hypoxic conditions or when stimulated with IL-1, IL-6, IL-17, IL-18, -prostaglandin, or TGF- $\beta$, or by CD40 ligation [2-4, 15-17]. Furthermore, VEGF knockout mice showed reduced pathology and synovial angiogenesis in antigen-induced models of arthritis [18]. These findings strongly suggest that the inhibition of the angiogenic action of VEGF is likely to suppress rheumatoid inflammation.

Angiogenesis and inflammation are interdependent processes, and inflammatory mediators have significant effects on angiogenesis [2, 3]. Furthermore, recent studies have suggested that the reverse is also true $[14,19]$. For example, chronic transgenic delivery of placental growth factor (PlGF) to murine epidermis resulted in a significant increase in inflammatory response [19]. In addition, in a previous study, we demonstrated that 165-amino acid form of VEGF, $\mathrm{VEGF}_{165}$, has a direct proinflammatory role in the pathogenesis of RA [14]. In this previous study, recombinant VEGF $_{165}$ was found to increase the productions of TNF$\alpha$ and IL- 6 by human peripheral blood mononuclear cells (PBMC). Moreover, the synovial fluid mononuclear cells of RA patients showed a greater response to $\mathrm{VEGF}_{165}$ stimulation than the PBMC of healthy controls (the major cell types that responded to VEGF were monocytes). These findings suggest that $\mathrm{VEGF}_{165}$ may act as a proinflammatory mediator and as an angiogenic stimulator in RA joints, and thus, they indicate that VEGF is an important link between angiogenesis and the inflammatory process.

A number of inflammatory cell types participate in maintaining a mutually activating network in RA joints, which leads to the establishment of a self-perpetuating

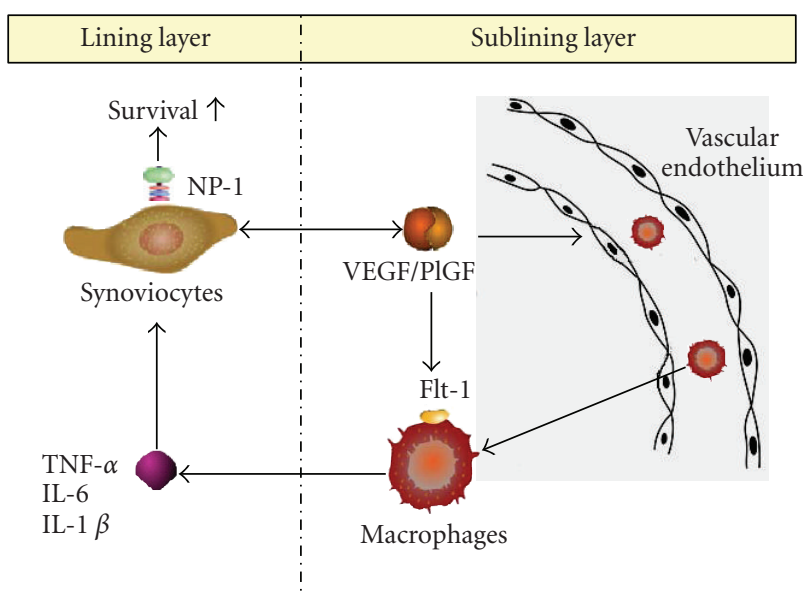

FIGURE 1: Scheme for the perpetuation of rheumatoid inflammation by VEGF. VEGF, which is predominantly produced by rheumatoid synoviocytes, promotes angiogenesis and stimulates vascular endothelial cell permeability. Newly employed macrophages produce TNF- $\alpha$ and IL- 6 when stimulated by VEGF/Flt-1 binding or by cell contact with activated endothelial cells. TNF- $\alpha$ and IL-6, in turn, further enhance the capacity of macrophages and synoviocytes to secrete VEGF, and thus create a self-perpetuating cycle of inflammation. Meanwhile, VEGF binding to NP-1 prevents rheumatoid synoviocytes undergoing apoptosis, which leads to synovial hyperplasia. Hyperplastic synoviocytes, in turn, secrete more $\mathrm{VEGF}_{165}$ and by so doing generate a positive feedbackloop that promotes survival. Thus, the development of synovial inflammation, hyperplasia, and angiogenesis in the joints of RA patients may be regulated by a common cue, VEGF.

cycle of autoimmunity [1]. It has been documented that VEGF $_{165}$ activates endothelial cells to produce chemokines, such as MCP-1 and IL-8 [20, 21], which may recruit monocytes around endothelial cells in synovial membranes, where newly employed macrophages, in addition to resident synoviocytes, can produce TNF- $\alpha$ and IL- 6 when stimulated by $\mathrm{VEGF}_{165}$ (as was evidenced by our work) or via cell contact with activated endothelial cells. TNF- $\alpha$ and IL-6, in turn, further enhance the capacities of macrophages and synoviocytes to secrete $\mathrm{VEGF}_{165}$, and stimulate endothelial cells to induce cell-contact-mediated macrophage activation, which generates a positive feedback-loop (Figure 1). Thus, VEGF $_{165}$ may serve as a functional bridge between endothelial cells and macrophages/synoviocytes.

In RA synovium, synovial fibroblasts proliferate abnormally and invade local environments, and in some ways exhibit the characteristics of tumor cells [22]. Recently, we demonstrated that VEGF is crucially required for the survival of rheumatoid synoviocytes [23]. In this previous study, the ligation of recombinant $\mathrm{VEGF}_{165}$ to its receptor prevented synoviocyte apoptosis induced by serum starvation or sodium nitroprusside (SNP). VEGF $_{165}$ rapidly triggered pAkt and pERK activity, and then induced $\mathrm{Bcl}-2$ expression in rheumatoid synoviocytes. Furthermore, VEGF 165 completely blocked SNP-induced Bcl-2 downregulation and SNPinduced Bax translocation from the cytosol to mitochondria. Collectively, these results suggest that VEGF functions as 
an important synoviocyte survival factor in RA. As mentioned above, $\mathrm{VEGF}_{165}$ is present at higher levels in sera, synovial fluid, and in the inflamed synovial tissues of RA patients than in those of osteoarthritis patients [13, 14]. Therefore, RA synoviocytes are more likely to be stimulated by VEGF $_{165}$ than osteoarthritis synoviocytes, which causes synoviocyte hyperplasia. Moreover, hyperplastic synoviocytes in RA joints secrete more $\mathrm{VEGF}_{165}$, and thus, generate positive feedback that promotes their survival (Figure 1).

\section{EXPRESSION AND FUNCTION OF VEGF RECEPTORS IN RA}

$\mathrm{VEGF}_{165}$ exerts its biological effects by binding with its receptor subtypes, that is, fms-like tyrosine kinase (Flt-1), kinase insert domain-containing receptor (KDR) and neuropilin-1 (NP-1) [3]. Flt-1 and KDR exhibit tyrosine kinase activity, and both are expressed in the majority of vascular endothelial cells $[8,24,25] . \mathrm{KDR}$ is a primary mediator of endothelial cell proliferation in response to $\mathrm{VEGF}_{165}$, whereas unlike KDR, Flt- 1 is present in inflammatory cells, such as, macrophages and monocytes $[8,24,25]$. Therefore, in addition to its proangiogenic action, Flt- 1 is critically involved in monocyte activation, and in addition, it also promotes the mobilization of myeloid progenitors from bone marrow to the blood $[8,24,25]$. On the other hand, NP-1 has been demonstrated to function as a nontyrosine kinase receptor for $\mathrm{VEGF}_{165}$, and specifically, for the heparin-binding domain of $\mathrm{VEGF}_{165}$ $[26,27]$. NP-1 was initially characterized as a receptor for semaphorin $3 \mathrm{~A}$, which mediates the guidance of neuronal cells [28]. In endothelial cells, NP-1 is also a coreceptor of VEGF, and has been shown to regulate KDR-dependent angiogenesis [26, 27]. Furthermore, NP-1 mediates the antiapoptotic activity of $\mathrm{VEGF}_{165}$ in breast cancer cells [29].

All three of $\mathrm{VEGF}_{165}$ receptor subtypes are expressed in RA synovium [30]. We previously demonstrated that NP-1, rather than Flt-1 or KDR, is the major VEGF $_{165}$ receptor in RA synoviocytes [23]. NP-1 was found to be highly expressed in the lining layer, and on infiltrating leukocytes and endothelial cells of the rheumatoid synovium. Furthermore, the downregulation of NP-1 transcripts by short interfering RNA caused spontaneous synoviocyte apoptosis, which was associated with both a decrease in Bcl-2 expression and an increase in Bax translocation to mitochondria. Therefore, NP-1 appears to play a critical role in maintaining RA synoviocyte survival. In addition, more recently, we found that Flt-1 is highly expressed in the sublining of leukocytes in RA synovium, and in monocytes from the PBMC of active RA patients [31]. Furthermore, Flt-1 expression levels were found to be well correlated with erythrocyte sedimentation rates, a marker of disease activity, indicating that they reflect inflammatory activity of RA [31]. These findings suggest that chronic inflammatory milieux, such as those generated by high concentrations of proinflammatory cytokines, may upregulate Flt-1 expression on RA monocytes.

There are several potential mechanisms whereby VEGF receptors could contribute to RA inflammation. First, $\mathrm{VEGF}_{165}$ binding to KDR may lead to an increase in angiogenesis, and thereby, the recruitment of peripheral leukocytes to inflamed synovium, which diminishes the growing burden of synoviocytes by supplying the oxygen and nutrients required for tissue metabolism. Second, via interaction with Flt-1, VEGF $_{165}$ may directly stimulate the productions of cytochemokines, such as TNF- $\alpha$, IL-6, MCP1 , and IL-8, which are essential for the perpetuation of chronic inflammation in joints. Third, NP-1 could hamper synoviocyte apoptosis upon ligation of $\mathrm{VEGF}_{165}$, and thus, function as a survival factor, in an autocrine or paracrine fashion. In this manner, $\mathrm{VEGF}_{165}$ would simultaneously regulate the developments of synovial inflammation, hyperplasia, and angiogenesis in RA joints (Figure 1).

\section{BLOCKADE OF VEGF AND ITS RECEPTOR IN RA}

The success of anti-VEGF antibody $(\mathrm{Ab})$ treatment in cancer patients raises the possibility of applying antiangiogenic therapies in other diseases, such as retinopathy, RA, and other inflammatory disorders. Given the pleiotropic roles played by VEGF and its receptor in RA inflammation [2, $3,8,24]$, it can be postulated that anti-VEGF treatment retards chronic synovitis in several ways, as follows: (a) it may decrease nutrient supply to the tumor-like synovium; (b) inhibit leukocyte adhesion and migration by decreasing endothelial cell surface area; (c) decrease chemokine and cytokine productions by activated endothelial cells; (d) reduce the VEGF-induced productions of TNF- $\alpha$ and IL6 by monocytes/macrophages; (e) abrogate VEGF-induced increases in synoviocyte survival. These different mechanisms may occur independently, but it remains to be determined which mechanism plays a dominant role in the quenching of RA inflammation.

Bevacizumab, a humanized form of anti-VEGF Ab, was the first antiangiogenic agent approved by the FDA in the US for the treatment of metastatic colon cancer [32], and it was also found to be beneficial for the treatment of lung and renal cell cancer $[33,34]$. Although bevacizumab is generally well tolerated, it has some serious toxic effects, for example, hypertension, bleeding, and arterial thromboembolism, which occur infrequently [32-34]. Currently, various different developmental approaches to inhibit VEGF and its receptors are in progress. The FDA approved pegaptanib, an anti-VEGF RNA aptamer, for the treatment of neovascular age-related macular degeneration (AMD) [35]. Notably, pegaptanib was found to reduce vision loss in AMD patients by about $50 \%$ during the first treatment year and to stabilize vision during the second year. Other agents which target VEGF receptors, such as, chimerized anti-KDR antibody, VEGF-Trap, and a synthetic ribozyme of Flt-1, are also undergoing phase I or II trials for the treatment of solid tumors and cancer [36].

The effects of VEGF and of its receptor antagonists have also been tested in experimental models of RA. Neutralizing $\mathrm{Ab}$ to VEGF was found to prevent collagen-induced arthritis and to ameliorate established disease in mice [37], and treatment with a soluble form of Flt-1 receptor significantly attenuated the severity of murine collagen-induced arthritis [38]. Interestingly, the failure of anti-KDR $\mathrm{Ab}$, but not of antiFlt-1 Ab, to block arthritis and atherosclerosis [24, 25, 39], 
indicates that anti-inflammation, rather than antiangiogenesis, may be primarily responsible for the observed effects of anti-VEGF Ab. Considering that Flt-1 tyrosine kinase signaling promotes RA via monocyte/macrophage activation $[31,40]$, the selective inhibition of Flt-1 may be effective at blocking VEGF-induced inflammation and angiogenesis with minimal toxicity. However, no clinical trial on antiVEGF inhibitors has been undertaken in RA. Indeed, some antirheumatic drugs with well-known clinical efficacy in RA, such as cyclosporin and anti-TNF- $\alpha \mathrm{Ab}$, have been reported to inhibit VEGF production in RA patients [41, 42].

Through the screening of positional scanning synthetic peptide libraries, we identified a soluble argininerich hexapeptide sequence, RRKRRR (Arg-Arg-Lys-Arg-ArgArg), which binds to $\mathrm{VEGF}_{165}$, and thereby prevents it from interacting with its receptor [14]. To increase the in vivo stability of this peptide, we changed its peptide structure from the L- to the D-form, and accordingly, were able to increase its half life to more than 24 hours [14], which makes the peptide more suitable for therapeutic applications. In mice, the hexapeptide RRKRRR significantly inhibited VEGF-induced angiogenesis, and also retarded the growth and metastasis of colon carcinoma cells. In addition, it strongly inhibited ongoing paw inflammation in arthritic mice without apparent side effects [14]. When compared with several known VEGF antagonists, such as anti-VEGF antibody and aptamer, RRKRRR is advantageous from the clinical standpoint because it is a short peptide that is easily synthesized and because it has low immunogenicity. In a similar manner, Bae et al., also found that the novel anti-Flt-1 hexapeptide, GNQWFI (Gly-Asn-Gln-Trp-PheIIe), inhibited angiogenesis and tumor growth without side effects [43]. This peptide selectively binds to Flt-1, and thereby, blocks the interaction between Flt-1 and VEGF or PlGF. Investigations on the effect of anti-Flt-1 peptide GNQWFI on an experimental model of arthritis are under way.

\section{PLACENTAL GROWTH FACTOR: A NEW POTENTIAL TARGET FOR ANTIANGIOGENIC TREATMENT}

PlGF is a member of the VEGF family, which was first identified in placenta, but is also known to be present in heart, lung, and joints [44]. As a specific ligand for Flt-1, PlGF has potent angiogenic properties, and it also induces the growth and migration of endothelial cells $[24,25]$. In addition, PlGF stimulates tissue factor production and chemotaxis in monocytes [45], and also increases TNF$\alpha$, IL-1, IL-6, IL-8, and MCP-1 productions by normal and/or rheumatoid monocytes [31, 46], which suggests that it directly modulates the inflammatory process. PlGF concentrations were reported to be increased in RA synovial fluids, and to induce VEGF production by mononuclear cells [47]. Moreover, genetic ablation of PlGF prevented the development of antibody-induced arthritis in mice [31] suggesting the critical role of PlGF in RA inflammation.

PlGF exhibits functions that are distinct from those of VEGF, in that it regulates the angiogenic switch during the diseased state [48]. It was recently reported that neutralizing
$\mathrm{Ab}$ to PlGF inhibits the growth and metastasis of various tumors, including those resistant to VEGF inhibitors, and that it enhances the efficacies of chemotherapy and that of anti-KDR Ab [48]. Unlike anti-KDR Ab, anti-PlGF Ab prevented the infiltration of angiogenic macrophages and severe tumor hypoxia, and thus, did not switch on the angiogenic rescue program responsible for resistance to antiKDR Ab [48]. Furthermore, it did not cause or enhance anti-KDR Ab-related side effects, such as, the inhibition of placental vascular development. Similar suppressive effects of PlGF antagonist on tumor growth were observed in another study, in which an antagonistic PlGF peptide (SHRYRLAIQLHASDSSSSCV) inhibited the growth and metastasis of human breast cancer xenografts [49]. Taken together, PlGF antagonists may prevent angiogenesis and tumor growth without affecting normal physiology, and thus, are ideal candidates for RA treatment. We are currently investigating whether synthetic anti-PlGF peptides inhibit the severity of arthritis and angiogenesis.

\section{CONCLUSION}

We and others have demonstrated that proangiogenic factors, such as VEGF and PlGF, exert direct proinflammatory $[14,19-21,31,40,45,46]$ and antiapoptotic effects [23, 29]. In this regards, the developments of synovial inflammation, hyperplasia, and angiogenesis in the joints of RA patients may all be regulated by VEGF. Given the importance of VEGF in the pathology of RA, antiangiogenic therapies, particularly those involving an anti-Flt-1 blocking agents, could when administered as a monotherapy or in combination with other biologic agents selectively ameliorate RA symptoms and reverse its fundamental pathology. The antiangiogenic peptides, RRKRRR and GNQWFI, introduced here represent a promising development in the antiangiogenic field. Hopefully, our efforts will result in clinical applications.

\section{ACKNOWLEDGMENTS}

This work was supported by Grants from the Ministry of Health and Welfare (South Korea) (no. A050196) and Korea Science and Engineering Foundation through Systems BioDynamics Research Center (R15-2004-033).

\section{REFERENCES}

[1] G. S. Firestein, "Evolving concepts of rheumatoid arthritis," Nature, vol. 423, no. 6937, pp. 356-361, 2003.

[2] A. E. Koch, "Angiogenesis: implications for rheumatoid arthritis," Arthritis \& Rheumatism, vol. 41, no. 6, pp. 951-962, 1998.

[3] N. Ferrara, H.-P. Gerber, and J. LeCouter, "The biology of VEGF and its receptors," Nature Medicine, vol. 9, no. 6, pp. 669-676, 2003.

[4] N. Maruotti, F. P. Cantatore, E. Crivellato, A. Vacca, and D. Ribatti, "Angiogenesis in rheumatoid arthritis," Histology and Histopathology, vol. 21, no. 4-6, pp. 557-566, 2006.

[5] V. Majithia and S. A. Geraci, "Rheumatoid arthritis: diagnosis and management," The American Journal of Medicine, vol. 120, no. 11, pp. 936-939, 2007. 
[6] R. O. Williams, E. Paleolog, and M. Feldmann, "Cytokine inhibitors in rheumatoid arthritis and other autoimmune diseases," Current Opinion in Pharmacology, vol. 7, no. 4, pp. 412-417, 2007.

[7] C. Salliot, M. Dougados, and L. Gossec, "Risk of serious infections during rituximab, abatacept and anakinra therapies for rheumatoid arthritis: meta-analyses of randomized placebocontrolled trials," Annals of the Rheumatic Diseases, vol. 68, pp. 25-32, 2009.

[8] M. Kowanetz and N. Ferrara, "Vascular endothelial growth factor signaling pathways: therapeutic perspective," Clinical Cancer Research, vol. 12, no. 17, pp. 5018-5022, 2006.

[9] J. D. Gorman, "Smoking and rheumatoid arthritis: another reason to just say no," Arthritis \& Rheumatism, vol. 54, no. 1, pp. 10-13, 2006.

[10] M. Raidl, B. Sibbing, J. Strauch, et al., "Impaired TNF $\alpha$ induced VEGF expression in human airway smooth muscle cells from smokers with COPD: role of MAPkinases and histone acetylation-effect of dexamethasone," Cell Biochemistry and Biophysics, vol. 49, no. 2, pp. 98-110, 2007.

[11] N. Rovina, A. Papapetropoulos, A. Kollintza, et al., "Vascular endothelial growth factor: an angiogenic factor reflecting airway inflammation in healthy smokers and in patients with bronchitis type of chronic obstructive pulmonary disease?" Respiratory Research, vol. 8, article 53, pp. 1-8, 2007.

[12] I. Edirisinghe, S.-R. Yang, H. Yao, et al., "VEGFR-2 inhibition augments cigarette smoke-induced oxidative stress and inflammatory responses leading to endothelial dysfunction," The FASEB Journal, vol. 22, no. 7, pp. 2297-2310, 2008.

[13] S.-S. Lee, Y.-S. Joo, W.-U. Kim, et al., "Vascular endothelial growth factor levels in the serum and synovial fluid of patients with rheumatoid arthritis," Clinical and Experimental Rheumatology, vol. 19, no. 3, pp. 321-324, 2001.

[14] S.-A. Yoo, D.-G. Bae, J.-W. Ryoo, et al., "Arginine-rich antivascular endothelial growth factor (anti-VEGF) hexapeptide inhibits collagen-induced arthritis and VEGF-stimulated productions of TNF- $\alpha$ and IL- 6 by human monocytes," The Journal of Immunology, vol. 174, no. 9, pp. 5846-5855, 2005.

[15] C.-S. Cho, M.-L. Cho, S.-Y. Min, et al., "CD40 engagement on synovial fibroblast up-regulates production of vascular endothelial growth factor," The Journal of Immunology, vol. 164, no. 10, pp. 5055-5061, 2000.

[16] S. Ryu, J. H. Lee, and S. I. Kim, "IL-17 increased the production of vascular endothelial growth factor in rheumatoid arthritis synoviocytes," Clinical Rheumatology, vol. 25, no. 1, pp. 16-20, 2006.

[17] M.-L. Cho, Y. O. Jung, Y.-M. Moon, et al., "Interleukin-18 induces the production of vascular endothelial growth factor (VEGF) in rheumatoid arthritis synovial fibroblasts via AP-1dependent pathways," Immunology Letters, vol. 103, no. 2, pp. 159-166, 2006.

[18] A. W. Mould, I. D. Tonks, M. M. Cahill, et al., "Vegfb gene knockout mice display reduced pathology and synovial angiogenesis in both antigen-induced and collagen-induced models of arthritis," Arthritis \& Rheumatism, vol. 48, no. 9, pp. 2660-2669, 2003.

[19] H. Oura, J. Bertoncini, P. Velasco, L. F. Brown, P. Carmeliet, and M. Detmar, "A critical role of placental growth factor in the induction of inflammation and edema formation," Blood, vol. 101, no. 2, pp. 560-567, 2003.

[20] T. Marumo, V. B. Schini-Kerth, and R. Busse, "Vascular endothelial growth factor activates nuclear factor- $\kappa \mathrm{B}$ and induces monocyte chemoattractant protein-1 in bovine retinal endothelial cells," Diabetes, vol. 48, no. 5, pp. 1131-1137, 1999.

[21] T.-H. Lee, H. Avraham, S.-H. Lee, and S. Avraham, "Vascular endothelial growth factor modulates neutrophil transendothelial migration via up-regulation of interleukin-8 in human brain microvascular endothelial cells," The Journal of Biological Chemistry, vol. 277, no. 12, pp. 10445-10451, 2002.

[22] G. S. Firestein, "Invasive fibroblast-like synoviocytes in rheumatoid arthritis: passive responders or transformed aggressors?" Arthritis \& Rheumatism, vol. 39, no. 11, pp. 17811790, 1996.

[23] W.-U. Kim, S. S. Kang, S.-A. Yoo, et al., "Interaction of vascular endothelial growth factor 165 with neuropilin-1 protects rheumatoid synoviocytes from apoptotic death by regulating Bcl-2 expression and Bax translocation," The Journal of Immunology, vol. 177, no. 8, pp. 5727-5735, 2006.

[24] A. Luttun, M. Tjwa, and P. Carmeliet, "Placental growth factor (PIGF) and its receptor Flt-1 (VEGFR-1): novel therapeutic targets for angiogenic disorders," Annals of the New York Academy of Sciences, vol. 979, pp. 80-93, 2002.

[25] M. Autiero, A. Luttun, M. Tjwa, and P. Carmeliet, "Placental growth factor and its receptor, vascular endothelial growth factor receptor-1: novel targets for stimulation of ischemic tissue revascularization and inhibition of angiogenic and inflammatory disorders," Journal of Thrombosis and Haemostasis, vol. 1, no. 7, pp. 1356-1370, 2003.

[26] S. Soker, S. Takashima, H. Q. Miao, G. Neufeld, and M. Klagsbrun, "Neuropilin-1 is expressed by endothelial and tumor cells as an isoform-specific receptor for vascular endothelial growth factor," Cell, vol. 92, no. 6, pp. 735-745, 1998.

[27] R. Mamluk, Z. Gechtman, M. E. Kutcher, N. Gasiunas, J. Gallagher, and M. Klagsbrun, "Neuropilin-1 binds vascular endothelial growth factor 165, placenta growth factor-2, and heparin via its b1b2 domain," The Journal of Biological Chemistry, vol. 277, no. 27, pp. 24818-24825, 2002.

[28] Z. He and M. Tessier-Lavigne, "Neuropilin is a receptor for the axonal chemorepellent semaphorin III," Cell, vol. 90, no. 4, pp. 739-751, 1997.

[29] R. E. Bachelder, A. Crago, J. Chung, et al., "Vascular endothelial growth factor is an autocrine survival factor for neuropilin-expressing breast carcinoma cells," Cancer Research, vol. 61, no. 15, pp. 5736-5740, 2001.

[30] M. Ikeda, Y. Hosoda, S. Hirose, Y. Okada, and E. Ikeda, "Expression of vascular endothelial growth factor isoforms and their receptors Flt-1, KDR, and neuropilin-1 in synovial tissues of rheumatoid arthritis," The Journal of Pathology, vol. 191, no. 4, pp. 426-433, 2000.

[31] H. S. Kim, S.-A. Yoo, H. J. Yoon, S. H. Park, C. S. Cho, and W.-U. Kim, "Role of placental growth factor and its receptor Flt-1 in rheumatoid inflammation," Arthritis \& Rheumatism. In press.

[32] N. Ferrara, K. J. Hillan, H.-P. Gerber, and W. Novotny, "Discovery and development of bevacizumab, an anti-VEGF antibody for treating cancer," Nature Reviews Drug Discovery, vol. 3, no. 5, pp. 391-400, 2004.

[33] J. C. Yang, L. Haworth, R. M. Sherry, et al., "A randomized trial of bevacizumab, an anti-vascular endothelial growth factor antibody, for metastatic renal cancer," The New England Journal of Medicine, vol. 349, no. 5, pp. 427-434, 2003.

[34] A. Sandler, R. Gray, M. C. Perry, et al., "Paclitaxel-carboplatin alone or with bevacizumab for non-small-cell lung cancer," 
The New England Journal of Medicine, vol. 355, no. 24, pp. 2542-2550, 2006.

[35] E. S. Gragoudas, A. P. Adamis, E. T. Cunningham Jr., M. Feinsod, and D. R. Guyer, "Pegaptanib for neovascular agerelated macular degeneration," The New England Journal of Medicine, vol. 351, no. 27, pp. 2805-2816, 2004.

[36] A. Kiselyov, K. V. Balakin, and S. E. Tkachenko, "VEGF/ VEGFR signalling as a target for inhibiting angiogenesis," Expert Opinion on Investigational Drugs, vol. 16, no. 1, pp. 83107, 2007.

[37] H. Sone, Y. Kawakami, M. Sakauchi, et al., "Neutralization of vascular endothelial growth factor prevents collageninduced arthritis and ameliorates established disease in mice," Biochemical and Biophysical Research Communications, vol. 281, no. 2, pp. 562-568, 2001.

[38] J. Miotla, R. Maciewicz, J. Kendrew, M. Feldmann, and E. Paleolog, "Treatment with soluble VEGF receptor reduces disease severity in murine collagen-induced arthritis," Laboratory Investigation, vol. 80, no. 8, pp. 1195-1205, 2000.

[39] M. De Bandt, M. H. Ben Mahdi, V. Ollivier, et al., "Blockade of vascular endothelial growth factor receptor I (VEGF-RI), but not VEGF-RII, suppresses joint destruction in the K/BxN model of rheumatoid arthritis," The Journal of Immunology, vol. 171, no. 9, pp. 4853-4859, 2003.

[40] M. Murakami, S. Iwai, S. Hiratsuka, et al., "Signaling of vascular endothelial growth factor receptor-1 tyrosine kinase promotes rheumatoid arthritis through activation of monocytes/macrophages," Blood, vol. 108, no. 6, pp. 1849-1856, 2006.

[41] M.-L. Cho, C.-S. Cho, S.-Y. Min, et al., "Cyclosporine inhibition of vascular endothelial growth factor production in rheumatoid synovial fibroblasts," Arthritis \& Rheumatism, vol. 46, no. 5, pp. 1202-1209, 2002.

[42] I. Macías, S. García-Pérez, M. Ruiz-Tudela, F. Medina, N. Chozas, and J. A. Girón-González, "Modification of pro- and antiinflammatory cytokines and vascular-related molecules by tumor necrosis factor- $\alpha$ blockade in patients with rheumatoid arthritis," The Journal of Rheumatology, vol. 32, no. 11, pp. 2102-2108, 2005.

[43] D.-G. Bae, T.-D. Kim, G. Li, W.-H. Yoon, and C.-B. Chae, "Anti-Flt1 peptide, a vascular endothelial growth factor receptor 1-specific hexapeptide, inhibits tumor growth and metastasis," Clinical Cancer Research, vol. 11, no. 7, pp. 26512661, 2005.

[44] J. DiSalvo, M. L. Bayne, G. Conn, et al., "Purification and characterization of a naturally occurring vascular endothelial growth factor. Placenta growth factor heterodimer," The Journal of Biological Chemistry, vol. 270, no. 13, pp. 77177723, 1995.

[45] M. Clauss, H. Weich, G. Breier, et al., "The vascular endothelial growth factor receptor Flt-1 mediates biological activities. Implications for a functional role of placenta growth factor in monocyte activation and chemotaxis," The Journal of Biological Chemistry, vol. 271, no. 30, pp. 17629-17634, 1996.

[46] S. K. Selvaraj, R. K. Giri, N. Perelman, C. Johnson, P. Malik, and V. K. Kalra, "Mechanism of monocyte activation and expression of proinflammatory cytochemokines by placenta growth factor," Blood, vol. 102, no. 4, pp. 1515-1524, 2003.

[47] M. J. Bottomley, N. J. A. Webb, C. J. Watson, et al., "Placenta growth factor (PIGF) induces vascular endothelial growth factor (VEGF) secretion from mononuclear cells and is co-expressed with VEGF in synovial fluid," Clinical \& Experimental Immunology, vol. 119, no. 1, pp. 182-188, 2000.
[48] C. Fischer, B. Jonckx, M. Mazzone, et al., "Anti-PlGF inhibits growth of VEGF(R)-inhibitor-resistant tumors without affecting healthy vessels," Cell, vol. 131, no. 3, pp. 463-475, 2007.

[49] A. P. Taylor and D. M. Goldenberg, "Role of placenta growth factor in malignancy and evidence that an antagonistic PIGF/Flt-1 peptide inhibits the growth and metastasis of human breast cancer xenografts," Molecular Cancer Therapeutics, vol. 6, no. 2, pp. 524-531, 2007. 


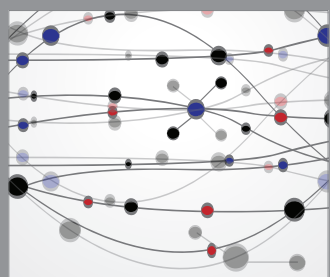

The Scientific World Journal
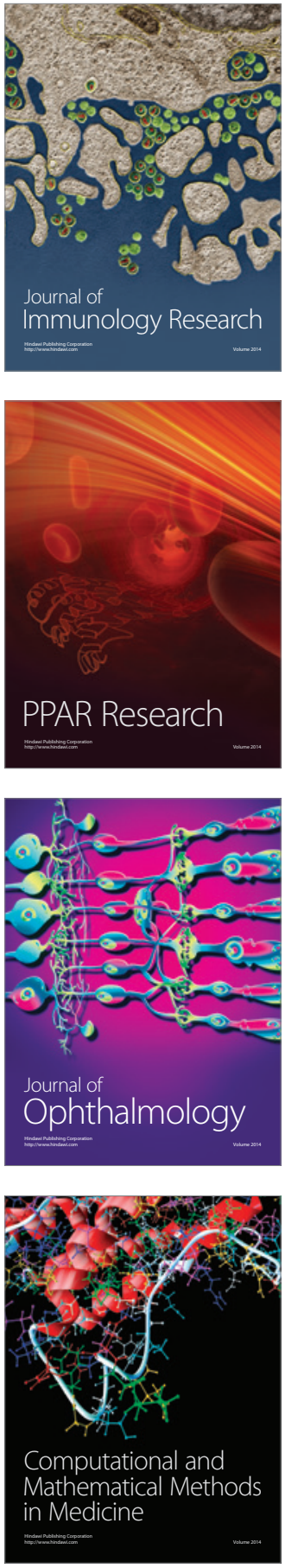

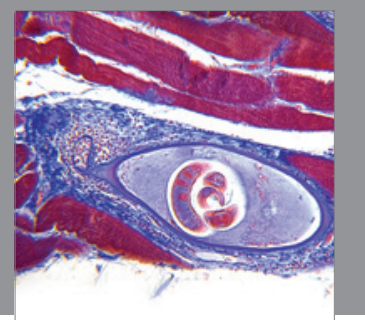

Gastroenterology

Research and Practice
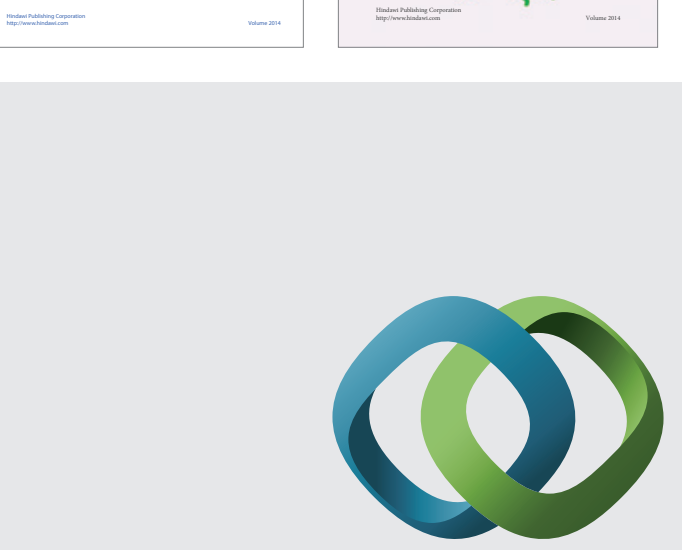

\section{Hindawi}

Submit your manuscripts at

http://www.hindawi.com
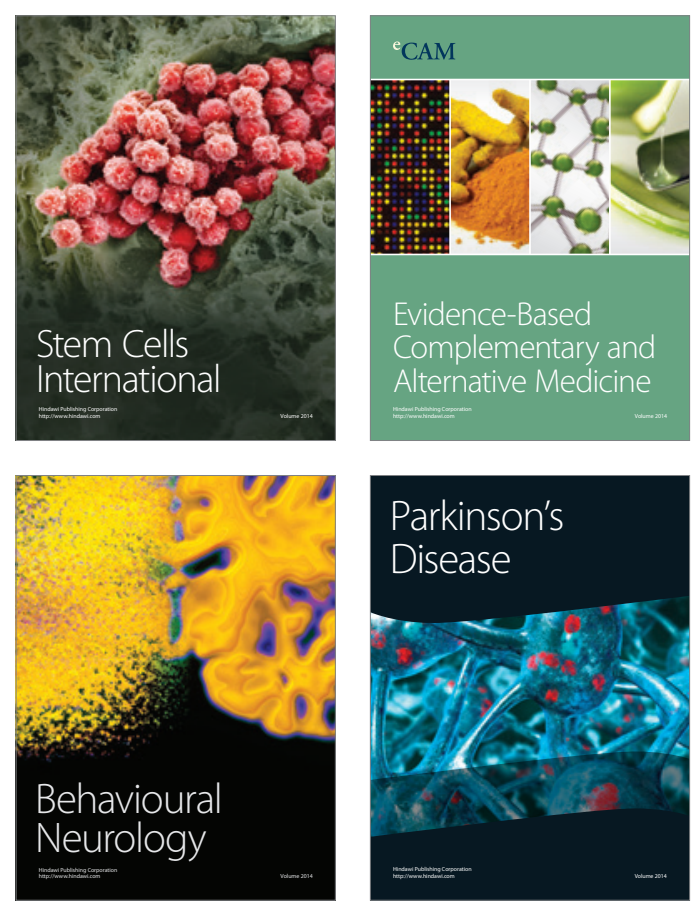

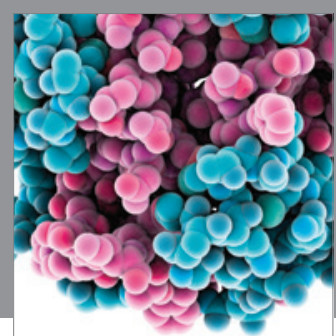

Journal of
Diabetes Research

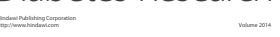

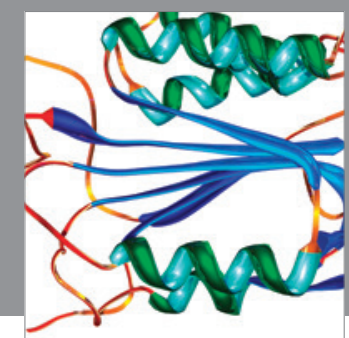

Disease Markers
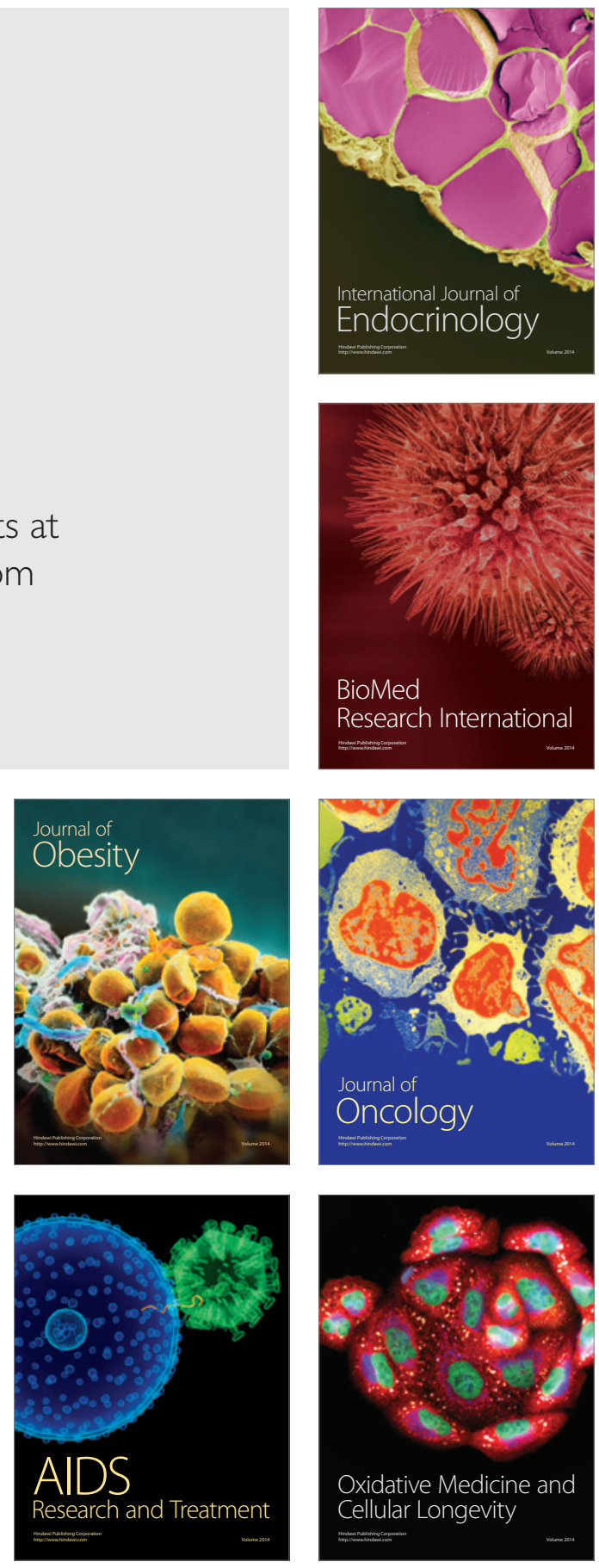